\title{
Center of Mass Estimation for Rehabilitation in a Multi-contact Environment: a Simulation Study
}

\author{
Alejandro González Mitsuhiro Hayashibe \\ INRIA \\ DEMAR team, LIRMM \\ Montpellier, France \\ Email: \{gonzalezde, hayashibe\}-at-lirmm.fr
}

\author{
Emel Demircan \\ Artificial Intelligence Laboratory \\ Stanford University \\ Palo Alto, California; USA \\ Email: emeld-at-stanford.edu
}

\author{
Philippe Fraisse \\ University of Montpellier 2 \\ DEMAR team, LIRMM \\ Montpellier, France \\ Email: fraisse-at-lirmm.fr
}

\begin{abstract}
Center of mass (CoM) estimation can be used to evaluate human stability during rehabilitation. A personalized estimation can be obtained using the serial equivalent static chain (SESC) method, calibrated using a series of static postures. The estimation accuracy is dependent on the number and quality of poses used during calibration. Currently, this limits the method's application to unimpaired individuals. We present a preliminary study of a SESC identified in a multi-contact scenario during a Sit-to-Stand task. Stanford's SAI (Simulation and Active Interface) platform was used to emulate motion and predict relevant reaction forces. The CoM estimation obtained is valid for motions similar to those used during identification. Using a threedimensional model, the estimated mean error was less than 26 millimetres for a Sit-to-Stand task involving displacements along all axes. As such, personalized CoM estimation can be available for patients with a limited range of whole body motion.

Index Terms - center of mass, parameter identification, operational space, Kalman filter, balance, multiple contacts, human balance
\end{abstract}

\section{INTRODUCTION}

The center of mass (CoM) trajectory is often used in humanoid robotics to generate stable locomotion. This is due to its close link to the concepts of center of pressure $(\mathrm{CoP})$ and zero moment point (ZMP). In human motion, CoM trajectory can be used to determine stability and to assess fall risks.

CoM position can be estimated by measuring the subject's pose and making use of anthropometric tables [1], [2] to approximate the mass and composition of each limb. This process, known as the kinematic method, is considered to be the golden standard [3], [4]. Since the information in the anthropometric tables was compiled for a given population, it does not accurately represent an individual from any other population. Gender, age, and fitness level affect the error of the CoM estimation. They play a part in the geometric and mass parameters of an individual [2], [5]; especially, those in need of rehabilitation could easily have a different mass distribution from that of the average individual. Accounting for these differences in order to improve the CoM estimation often requires a trained clinician to perform a long set of measurements, or the use of expensive medical imaging equipment [5].

To overcome this limitation the statically equivalent serial chain (SESC) was introduced by Cotton et al. [6] as a tool for 978-1-4799-0652-9/13/\$31.00 (C) 2013 IEEE

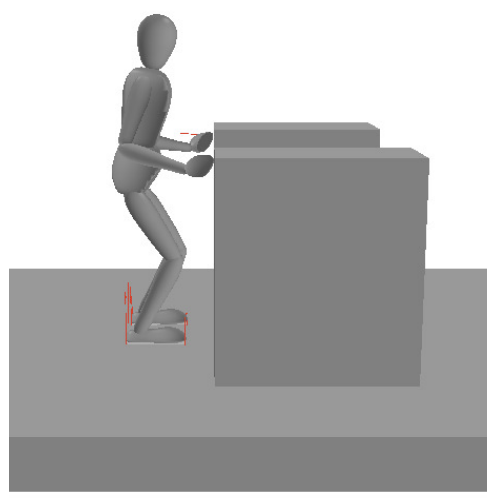

Fig. 1. The simulation environment SAI is used to reproduce a common situation during balance training: standing with multiple supporting contacts. During simulation the position of the human model's hands and total CoM position are controlled. At the same time, contact forces can be obtained and used for SESC identification.

CoM estimation in humans, based on the work of Espiau and Boulic [7]. After the method was validated for the control of humanoid robots, Cotton et al. [6] used it to estimate the CoM position of a young individual on the sagittal plane. A later study applied the SESC to a group of elderly volunteers and compared its performance to that of other methods [8]. Like the kinematic method, the SESC's personalized CoM estimation is dependent on joint angular measurements. This makes it suitable for the analysis of dynamic motions.

The calibration method proposed by [6] uses CoP information during static poses. It suggests that a large number of measurements, in distinct configurations, is required to increase the chain's accuracy. In our experience, the calibration procedure may be long and physically demanding. This prevents the application of the SESC on individuals suffering from a balance impairment or a having a high fall risk. With this in mind, we have studied the SESC when calibrated in a on-line manner [9], seeking to reduce the physical demands of the identification procedure. It is also possible to offer real-time feedback to the patient to decrease calibration time. Nonetheless, the flexibility achieved with a Kalman filter calibration does not address the problem of multiple contact 
points allowing the subject to fully support his weight. This is a common situation on during rehabilitation training and has prompted the current study.

We propose to study the identifiability of the SESC parameters in a multi-contact situation when forces are measured at each contact point. We seek to approximate the conditions of a patient who needs the aid of a walker. That is, our goal is to obtain a subject-specific CoM estimate for patients who require multiple supporting contact points during standing or walking. As in [9], a constrained linear Kalman filter is used. Both data selection, and parameters estimation are done during run-time.

In the context of the @WALK project, we make use of the robotics platform SAI [10] to simulate the dynamic behaviour of a human model. Body trajectories were generated to roughly resemble those of a patient during a rehabilitation session. Ground reaction forces and limb orientations are measured in SAI and used as input for the recursive SESC calibration. Also, since the description of all links is exactly known, the model's CoM position is available. It will serve as the ground truth to which the calibrated SESC will be evaluated.

\section{Method}

We make use of SAI (Simulation and Active Interface developed by the Artificial Intelligence Laboratory at Stanford University) [10] to simulate the behaviour of a human model. SAI was originally designed to accurately simulate the dynamics of robots with high degrees of freedom. With it, we are able to visualize the influence that multiple contact forces have in our model. Fig. 1 represents a common situation for subjects performing balance training exercises, such as patients recovering from neurovascular injuries like a stroke, or elderly subjects who require the aid of a cane or walker for ambulation.

The human model, Stanbot, was controlled using Khatib's operational space formulation [11]. Task prioritization is possible in this framework using the null space of the corresponding task Jacobian. Details for this will be given in Sec. II-A.

Contact forces were calculated and used to estimate the model's center of pressure (CoP). They were also used to determine if the model was indeed in static equilibrium. This static CoP estimate was then used as input to identify the geometric parameters of a statically equivalent serial chain (SESC). Further details regarding the identification/validation trajectories are given in Sec. III

\section{A. Operational Space Framework}

A robot's end effector position can be controlled in the joint space by applying a torque to each joint. Khatib [11] presented an alternative to this in which the motion of the end effector, known as the operational point, is defined in terms of tasks. Each task is in turn described by the robot's dynamics and by the forces acting on the operational point. This is known as the operational space.

The dynamics of the operational point are described by the second order differential equation:

$$
\Lambda(x) \ddot{\vec{x}}+\vec{\mu}(\dot{\vec{x}}, \vec{x})+\vec{p}(\vec{x})=\vec{F}
$$

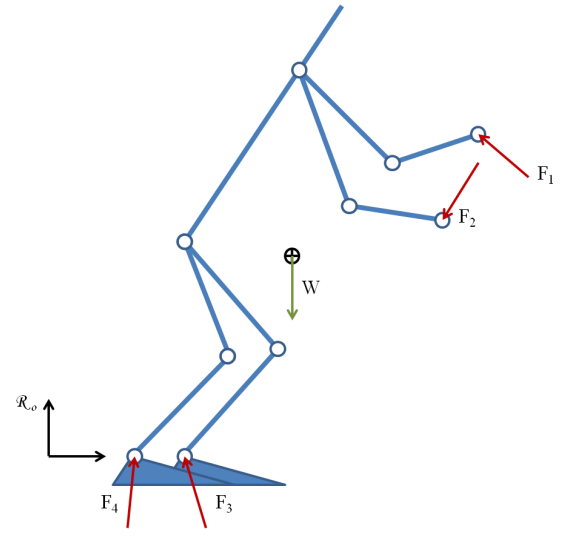

Fig. 2. Free body diagram of a tree-structured chain with multiple contacts. For the static case, it is possible to determine the ground projection of the chain's CoM when all the contact forces and their application points are known.

where $\vec{x}$ represents the position of the operational point, $\boldsymbol{\Lambda}$ can be thought of as a kinetic energy matrix, $\vec{\mu}$ is a vector composed of Coriolis and centrifugal forces, and $\vec{p}$ contains the gravity forces. When the dynamic model of the robot is known, it is possible to calculate the force vector $\vec{F}$ that will be applied to the operational point. Control torques $(\boldsymbol{\Gamma})$ can then be reported to joint level by the use of the corresponding task Jacobian (J).

$$
\Gamma=\mathbf{J}^{T} \vec{F}
$$

Redundant degrees of freedom can be used up by performing additional tasks. Ideally, each task is performed with no adverse effects on any other task; this is achieved by prioritization. A second priority task is only performed when it does not interfere with a higher priority task. This sequencing can be described using the higher priority task's the null space: $\left(\mathbf{I}-\mathbf{J}^{T} \overline{\mathbf{J}}^{T}\right)$. The torque vector can then be composed as:

$$
\boldsymbol{\Gamma}=\mathbf{J}^{T} \vec{F}+\left(\mathbf{I}-\mathbf{J}^{T} \overline{\mathbf{J}}^{T}\right) \vec{\Gamma}_{0}
$$

where $\overline{\mathbf{J}}$ is a generalized inverse of the task's Jacobian, $\vec{\Gamma}_{0}$ represents a second task that will be accomplished if it does not affect the first one, and $\mathbf{I}$ is a suitable identity matrix.

The definition of $\overline{\mathbf{J}}$ dictates the manner in which the prioritization is performed. In this work we use the dynamically consistent generalized inverse of the Jacobian [11].

\section{B. Motion Control}

Each task is controlled by setting a control vector, $\vec{F}^{*}$, for the operational point [11]. This acceleration will be the input of the dynamic equation (1). We wish to control i) the position of the model's hands, and ii) CoM trajectory. This can be considered as the definition of 3 tasks: one for the $\mathrm{CoM}$ and one for each hand. We choose to implement a PD controller to track the desired trajectories of each operational point. The controllers will be defined by the values $k_{v}$ and $k_{p}$, representing the derivative and proportional gains respectively. Hand position was specified as given by (4)

$$
\vec{F}^{*}=-k_{v} \dot{\vec{x}}-k_{p}\left(\vec{x}-\vec{x}_{d}\right)
$$



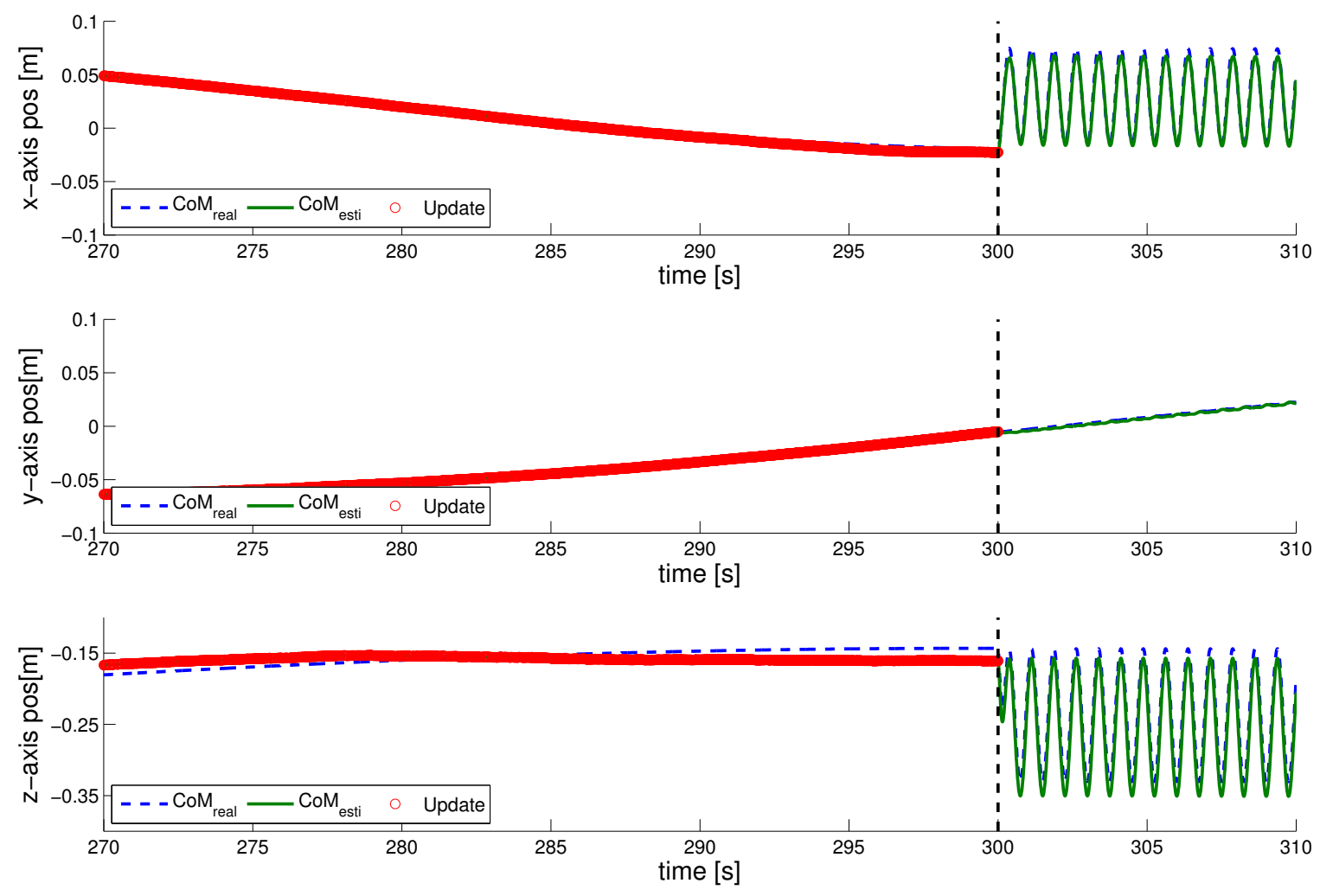

Fig. 3. Estimated CoM is shown with a solid green line. The model's CoM, the ground truth, shown here by a dashed blue line is given for comparison purposes. The SESC parameters are no longer updated after 300 seconds. Using the Kalman filter, each update improves our CoM estimate. This can be seen by the eventual convergence of the green and blue lines.

with $\vec{x}_{d}$ as the operational point's desired position. CoM trajectory was controlled by (5), which includes the operational point's desired velocity and acceleration, $\dot{\vec{x}}_{d}$ and $\ddot{\vec{x}}_{d}$.

$$
\vec{F}^{*}=\ddot{\vec{x}}_{d}-k_{v}\left(\dot{\vec{x}}-\dot{\vec{x}}_{d}\right)-k_{p}\left(\vec{x}-\vec{x}_{d}\right)
$$

An additional low priority task was added to drive the model to a neutral posture: far from joint limits. This posture, reminiscent of quiet standing, was calculated in the joint space and was defined by the input torque $\vec{\Gamma}_{0}$.

\section{Statically Equivalent Serial Chain (SESC)}

The CoM of any linked chain can be represented as the end effector of a statically equivalent serial chain (SESC) [6]. The SESC is described by a group of parameters $(\vec{R})$ which describe the geometry of its links. $\vec{R}$ can be calculated from explicit knowledge of the original chain's geometry and the masses of its links. Using the SESC method, the chain's CoM, $\vec{C}_{M}$, may be expressed as the matrix multiplication:

$$
\vec{C}_{M}=\mathbf{B} \vec{R}
$$

where $\mathbf{B}$ is assembled with the direction cosine matrix of the chain's links. This method holds for all motions in which the segment orientation can be measured.
When it is not possible to define $\vec{R}$ due to lack of knowledge of the linked chain, SESC parameters can be approximated via geometric calibration. The procedure uses a series of joint configurations for which the CoM is known; at least partially. After enough measurements have been performed, $\vec{R}$ can be estimated in the least-squares sense using the MoorePenrose pseudo inverse [6], [12]. A suitable estimate can also be obtained on-line by a recursive approach such as the Kalman Filter [9]. Details for the explicit calculation and estimation of $\vec{R}$ can be found in [6], [12].

\section{Kalman Filter}

Consider the linear, time variant system:

$$
\vec{Y}_{k}=\mathbf{H}_{k} \vec{X}_{k}+\vec{v}_{k}
$$

This system is, by definition, a linear combination of the state vector $\vec{X}_{k}$ and a zero mean noise $\vec{v}_{k}$ with a known covariance $\mathbf{R}_{k} . \mathbf{H}$ is known as the configuration matrix.

It is possible to estimate $\vec{X}_{k}$ by performing a measurement $\vec{Y}_{k}$ at time $t_{k}$. Each new measurement improves our knowledge of the state vector. Among the recursive state estimators, the Kalman filter offers is optimal for system (7).

A special case of the Kalman filter can be used when $\vec{X}$ is constant and subject to a zero mean noise of covariance 
$\mathbf{Q}_{k}$ [13], [14]. The filter equations may be written as follows:

$$
\begin{aligned}
\mathbf{P}_{k}^{-} & =\mathbf{P}_{k-1}^{+}+\mathbf{Q}_{k-1} \\
\mathbf{K}_{k} & =\mathbf{P}_{k}^{-} \mathbf{H}_{k}^{T}\left(\mathbf{H}_{k} \mathbf{P}_{k}^{-} \mathbf{H}_{k}^{T}+\mathbf{R}_{k}\right)^{-1} \\
\hat{\vec{X}}_{k} & =\overrightarrow{\vec{X}}_{k-1}+\mathbf{K}_{k}\left(\vec{Y}_{k}-\mathbf{H}_{k} \overrightarrow{\vec{X}}_{k-1}\right) \\
\mathbf{P}_{k}^{+} & =\left(\mathbf{I}-\mathbf{K}_{k} \mathbf{H}_{k}\right) \mathbf{P}_{k}^{-}
\end{aligned}
$$

where $\mathbf{P}_{k}^{-}$and $\mathbf{P}_{k}^{+}$are the estimation covariances before and after the state update, $\mathbf{I}$ is an identity matrix of suitable size, and $\mathbf{K}_{k}$ is a optimal filter gain. This filter will find the optimal state vector in the least squares sense.

\section{E. Skeletal model definition}

For the purpose of CoM estimation using a SESC, a skeletal model with nine thin, rigid links was used (see Fig. 2). One link represents the torso, pelvis, and head. The others; the left/right upper and lower arms, and the left/right upper and lower legs. All links are joined by spherical joints. Hands and feet were neglected due to their small contribution to CoM position.

Excepting the torso, the CoM of all links was assumed to lie on the line connecting its two extremes. The SESC associated to such a model can be described using seven parameters [9].

\section{F. Multi-contact situation}

During the SESC calibration phase it is necessary to have access to at least one component of CoM position. Previous studies [6], [8], [9], [12] have used a force platform to measure $\mathrm{CoP}$ position for a number of static poses. This provides two of the three needed components for estimating $\vec{R}$ of a threedimensional SESC.

$\mathrm{CoP}$ measurement with a force platform is only valid when all contact points are inside the sensing area. This prevents the calibration procedure, in its present form, from being applied on patients who require additional supporting contacts. We wish to study the computational feasibility of SESC identification under the multi-contact condition. We assume that all contact forces are known. They can be measured, for example, by the inclusion of force sensors at the appropriate places in walkers or other supporting structures.

Consider the chain depicted in Fig. 2. We assume that: a) all forces acting on the chain are known, and b) that $\sum \vec{F}=0$; that is, the CoM is not being accelerated during the identification phase. It is possible to determine the projection of the chain's CoM to the ground using (12):

$$
\vec{C}_{M}=\frac{\vec{n} \times \vec{M}_{o}}{\vec{W} \cdot \vec{n}}
$$

where $\vec{M}_{o}$ is the sum of moments around the origin caused by the reaction forces, $\vec{W}$ is the chain's weight, and $\vec{n}$ is a unit vector normal to the ground surface. This notation is a special case of the equation used by Sardain and Bessonet [15] for estimating the position of the $\mathrm{CoP}$ in a flat surface. We note that, for static cases, the position of the CoP corresponds to the ground projection of the chain's CoM.

\section{Simulation STUdY}

During simulation, we approximated the range of motion of an impaired individual in a multi-contact scenario. This was achieved by controlling the posture of a human analog using SAI (see Fig. 1). Three tasks were prioritized using the operation space framework. In descending priority order, we controlled: 1) Hand position to be in constant contact with the environment. 2) The CoM trajectory. The CoM's motion in all three axes was decoupled. A sine function served as input for each direction. Sinusoidal trajectories were used to provide a simple rhythmical motion that could be followed by a real patient. 3) Joint configuration to simulate quiet standing. Controller gains were tuned empirically in order to obtain a fast response from the model. Reaction forces were obtained from the simulation's contact model and used to estimate the ground projection of CoM.

Using a Kalman filter we estimated the parameters of the SESC whose end effector will reproduce the motion of the human model's CoM. Measurements were discriminated by observing the sum of the forces acting on the human model. A measurement was used to update the chain's estimate only when this sum approaches zero; that is, close to the quasistatic condition. This assured that our measurement accurately reflected the ground projected CoM position.

A nine segment SESC was used to estimate CoM position. Details of the skeletal model were given in Sec. II-E. The orientation of each segment corresponds to that of the lower and upper legs, the arm and forearms, and trunk segments. Two simulations were performed: A. used for SESC identification, and $\mathrm{B}$. used to validate the CoM estimation by performing a three-dimensional motion.

\section{A. SESC Identification}

The human analog motion's were created with an impaired human patient in mind. A volume inside which the CoM could move was created by tuning low amplitude sine patterns in each direction. The allowed CoM oscillation had a peak-to-peak amplitude of 100 millimetres front-back with a 200 second period, 140 millimetres left/right with a 321 second period and of 100 millimetres up-down with an 347 second period. The oscillation periods were chosen avoiding multiples of each other to increase data dispersion. The experiment ran for 300 seconds in-simulation time after which the Kalman filter was disabled. The period of oscillation in the $x z$-plane was reduced and CoM position was estimated using the latest parameter values during 60 seconds. This was done to show the validity of the SESC estimation for different speed motions.

\section{B. Three-dimensional motion}

Using the previously identified SESC model, we estimated the trajectory of the CoM while it moved in three dimensions. The motion along was generated by modifying the period of oscillation of the decoupled CoM components while maintaining their amplitude. The model's contacts to the environment were also maintained. 

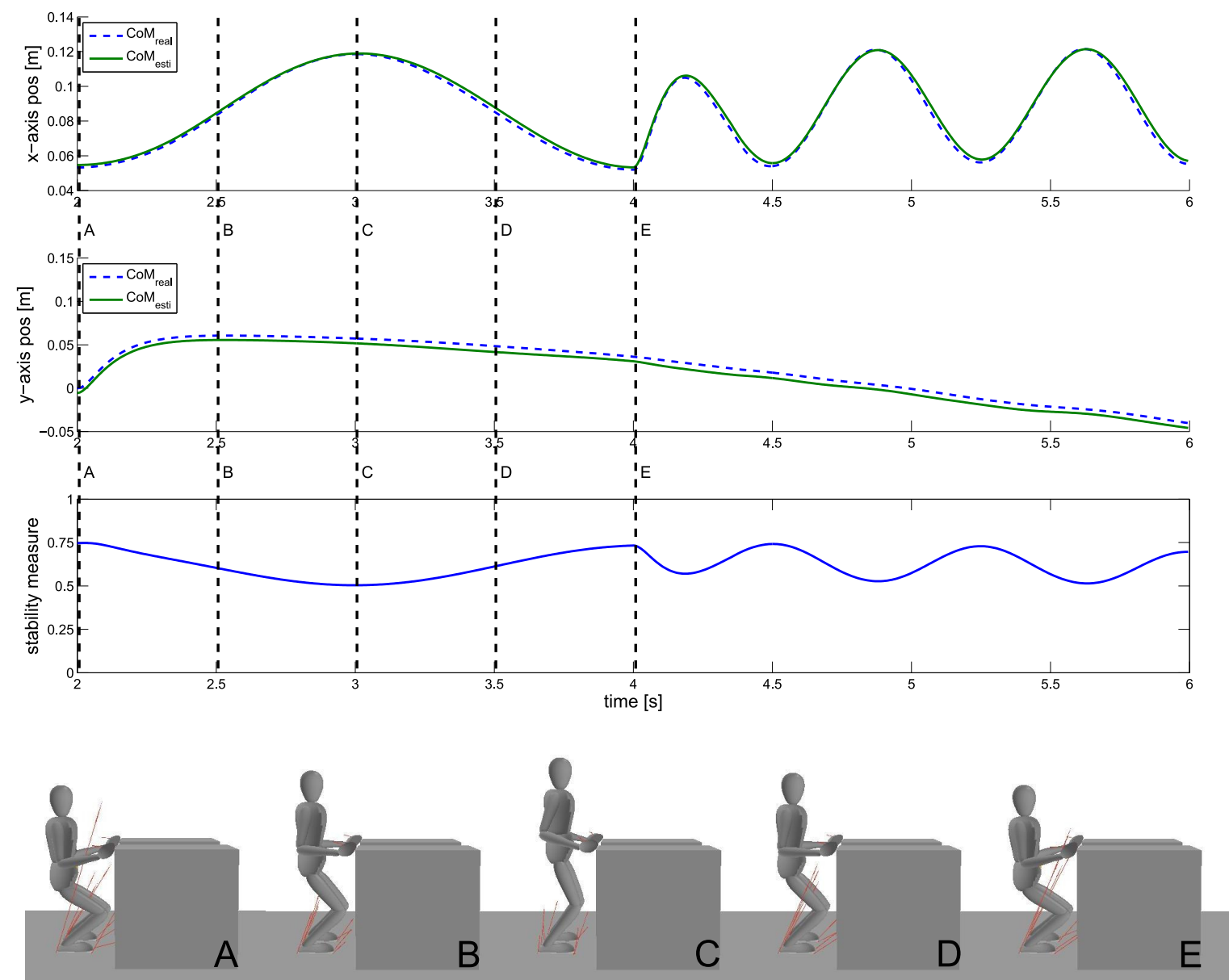

Fig. 4. Measured and estimated CoM position of a Sit-to-Stand task. The human model stands up and sits back down in two seconds. Intermediate poses are shown (labelled A-E) and related to the CoM position along the motion. Correct tracking is obtained regardless of the movement speed, as the CoM estimate only requires measuring the limb orientation. The last plot shows a stability measured based on the need of taking a step (value of zero) in order to maintain the CoM inside the region of support.

The motion performed is reminiscent of a Sit-to-Stand transfer but presents a simplified movement. A slow displacement of the CoM along the direction of the medio-lateral axis was included to show the three-dimensional estimate The trajectory performed during this test; although different to the ones used for identification, maintains a similar orientation of the lower arms and legs.

\section{RESUlTS}

Results are reported as: 1) the root mean squared error (rmse) of the SESC estimate to real CoM position for data not used during identification, 2) their relative standard error (rse), calculated as follows:

$$
\text { rse }=100 \% \cdot\left(\frac{\sigma_{e}}{\sqrt{n}}\right) \cdot\left(\frac{1}{\bar{e}}\right)
$$

where $\vec{e}$ is the error vector $\vec{e}=\vec{C}_{e s t}-\vec{C}_{M}, \sigma_{e}$ indicates the vector's standard deviation, $\bar{e}$ its mean, and $n$ its length., and 3) the average three-dimensional distance between the real and estimated CoM position.

\section{A. SESC Identification}

Fig. 3 shows the result of the simulation. The CoM actual position is shown using a dashed blue line. It was calculated by the SAI software from the complete knowledge of the model's geometry and mass. The model's estimated CoM position is shown with the solid green line. It was calculated using the most recent parameters estimate and the current segment orientation.

The average distance between the ground projections of the real and estimated CoM, after identification was achieved, was 6.09 millimetres. Table I shows the rmse and rse for two validation sets: one with the CoM inside the identification volume, the other for a Sit-to-Stand task.

\section{B. Three-dimensional motion}

The CoM estimate obtained using the SESC method is shown with a solid green line in Fig. 4. On the same figure we show the CoM position, in blue, as calculated with SAI. The mean distance error of the CoM ground projection was found to be of 7.86 millimetres. The obtained estimation errors can 
TABLE I

ESTIMATION ERROR

\begin{tabular}{|c|c|c|c|c|c|c|}
\hline & \multicolumn{3}{|c|}{ ID Volume ${ }^{a}$} & \multicolumn{3}{|c|}{ Sit-to-Stand ${ }^{b}$} \\
\hline & $x$ & $y$ & $z$ & $x$ & $y$ & $z$ \\
\hline rmse $[\mathrm{mm}]$ & 5.61 & 3.64 & 18.67 & 9.08 & 5.53 & 24.80 \\
\hline rse [\%] & 0.82 & 0.88 & 0.15 & 13.92 & 0.95 & 0.87 \\
\hline distance $[\mathrm{mm}]$ & & 19.65 & & & 25.68 & \\
\hline
\end{tabular}

We evaluate the CoM estimation error when the Filter updates are disabled: ${ }^{a}$ shows the errors for CoM movement similar to those performed during identification, while ${ }^{b}$ shows the estimation error for a three-dimensional Sit-to-Stand task.

be found in Table I

\section{DISCUSSION AND CONCLUSION}

The SESC model is highly redundant with respect to the CoM position. That is why the accuracy of its estimation is closely related to the variation of the information available during the identification step. Though not an unique solution, the best SESC parameters are found after processing data in a large range of the subject's possible motions [6]. This is an important limitation of the identification method; preventing it from being used with balance impaired subjects. Simply stated: if the subject is not capable of performing a large range of identification poses, the subject-specific SESC may not be available. For this reason, we evaluated the SESC estimation when its parameters were identified under these circumstances. Even using a small range of different poses, a SESC can be calibrated and used to estimate CoM position. Estimation errors may occur when moving away from the supposed range of motion. For example, the small variation of arm orientation during the identification may have caused its influence on the total CoM position to be overestimated. If the arms are moved, the SESC estimation could no longer be valid. The experimental data should be given uniformly over the subject's supposed range of motion in order to obtain a good model. As long as this requirement is fulfilled, we claim that the SESC identification is feasible even in the multi-contact environment and with a limited range of motion.

For the poses used, we note a relatively fast convergence time, close to five minutes. A good tracking of the CoM ground truth was performed after the filter updates are stopped. It is important to emphasize that in spite of its name, the SESC method for CoM estimation can still be valid during dynamic motions. After the calibration phase is performed, only the subject's limb orientations are necessary to estimate CoM position. The quasi-static condition is only required during the identification step of the SESC parameters. Figures 3 and 4 support this, as we observe a good match of the estimated $\mathrm{CoM}$ and ground truth positions even for motions performed at different speeds.

A good CoM estimate can be used reliably assess the patient's balance. For example, Fig. 4 shows a measure which approaches zero as the CoM projection leaves the area which can be covered by taking a single step. That is, if the CoM can still be brought to rest by taking only one step. This is loosely based on the concept of the zero rate of change of angular momentum (ZRAM) [16].

\section{ACKNOWLEDGEMENTS}

This work is supported by the @WALK (Artificial WALKing), a part of INRIA's "Associate Teams" program.

We wish to thank Jinsung Kwon and Francois Conti for their help to understand and develop controllers with the SAI simulator. Also, many thanks to Prof. Khatib and all the members of Stanford's Artificial Intelligence Laboratory for their kind welcome and hospitality during the development of this work.

\section{REFERENCES}

[1] D. A. Winter, Biomechanics and motor control of human movement. Hoboken, N.J.: John Wiley \& Sons, Inc, 2005.

[2] P. de Leva, "Adjustments to Zatsiorsky-Seluyanov's segment inertia parameters," Journal of Biomechanics, vol. 29, no. 9, pp. 1223-1230, Sep. 1996.

[3] D. Lafond, M. Duarte, and F. Prince, "Comparison of three methods to estimate the center of mass during balance assessment." Journal of biomechanics, vol. 37, no. 9, pp. 1421-1426, Sep. 2004.

[4] A. L. Hof, "Comparison of three methods to estimate the center of mass during balance assessment." Journal of biomechanics, vol. 38, no. 10, pp. 2134-5, Oct. 2005.

[5] M. A. Jaffrey, "Estimating Centre of Mass Trajectory and SubjectSpecific Body Segment Parameters Using Optimisation Approaches," PhD, Victoria University, 2008.

[6] S. Cotton, A. P. Murray, and P. Fraisse, "Estimation of the Center of Mass: From Humanoid Robots to Human Beings," IEEE/ASME Transactions on Mechatronics, vol. 14, no. 6, pp. 707-712, Dec. 2009.

[7] B. Espiau and R. Boulic, "On the Computation and control of the mass center of articulated chains," Grenoble, France, 1998.

[8] S. Cotton, M. Vanoncini, P. Fraisse, N. Ramdani, E. Demircan, A. P. Murray, and T. Keller, "Estimation of the centre of mass from motion capture and force plate recordings : a study on the elderly." Applied Bionics and Biomechanics, vol. 8, no. 2, pp. 67-84, 2011.

[9] A. González, M. Hayashibe, and P. Fraisse, "Online Identification and Visualization of the Statically Equivalent Serial Chain via Constrained Kalman Filter," in IEEE International Conference on Robotics and Automation (ICRA), Karlsruhe [Germany], 2013, pp. 5303-5308.

[10] O. Khatib, O. Brock, K.-S. Chang, F. Conti, D. Ruspini, and L. Sentis, "Robotics and interactive simulation," Communications of the ACM, vol. 45, no. 3, pp. 46-51, 2002.

[11] O. Khatib, "A unified approach for motion and force control of robot manipulators: The operational space formulation," IEEE Journal of Robotics and Automation, pp. 43-53, 1987.

[12] A. González, M. Hayashibe, and P. Fraisse, "Estimation of the Center of Mass with Kinect and Wii balance board," in IEEE/RSJ International Conference on Intelligent Robots and Systems (IROS), Vilamoura, Alagarve [Portugal], 2012, pp. 1023-1028.

[13] D. Simon, Optimal State Estimation: Kalman H Infinity, and Non Linear Approaches. John Wiley \& Sons, Inc, 2006.

[14] B. W. Mooring, Z. S. Roth, and M. R. Driels, Fundamentals of Manipulator Calibration. Wiley Press, 1991.

[15] P. Sardain and G. Bessonnet, "Forces Acting on a Biped Robot. Center of Pressure - Zero Moment Point," IEEE Transactions on Systems, Man, and Cybernetics - Part A: Systems and Humans, vol. 34, no. 5, pp. 630-637, Sep. 2004.

[16] A. Goswami and V. Kallem, "Rate of Change of Angular Momentum and Balance Maintenance of Biped Robots," in IEEE International Conference on Robotics and Automation (ICRA), New Orleans, Lousianna [USA], 2004, pp. 3785-3790. 